\title{
Denoising arterial spin labeling MRI using tissue partial volume
}

\author{
Jan Petr ${ }^{1,3,4}$, Jean-Christophe Ferré ${ }^{1,2,3,4}$, Jean-Yves Gauvrit ${ }^{1,2,3,4}$ and Christian Barillot ${ }^{1,3,4}$ \\ ${ }^{1}$ INRIA, VisAGeS Project-Team, F-35042 Rennes, France \\ ${ }^{2} \mathrm{CHU}$, University Hospital of Rennes, Radiology Dept., F-35043 Rennes, France \\ ${ }^{3}$ University of Rennes I, CNRS, UMR 6074, IRISA, F-35042 Rennes, France \\ 4INSERM, U746, F-35042 Rennes, France
}

\begin{abstract}
Arterial spin labeling (ASL) is a noninvasive MRI method that uses magnetically labeled blood to measure cerebral perfusion. Spatial resolution of ASL is relatively small and as a consequence perfusion from different tissue types is mixed in each pixel. An average ratio of gray matter (GM) to white matter (WM) blood flow is 3 to 1. Disregarding the partial volume effects (PVE) can thus cause serious errors of perfusion quantification. PVE also complicates spatial filtering of ASL images as apart from noise there is a spatial signal variation due to tissue partial volume. Recently, an algorithm for correcting PVE has been published by Asllani et al. It represents the measured magnetization as a sum of different tissue magnetizations weighted by their fractional volume in a pixel. With the knowledge of the partial volume obtained from a high-resolution MRI image, it is possible to separate the individual tissue contributions by linear regression on a neighborhood of each pixel. We propose an extension of this algorithm by minimizing the total-variation of the tissue specific magnetization. This makes the algorithm more flexible to local changes in perfusion. We show that this method can be used to denoise ASL images without mixing the WM and GM signal.
\end{abstract}

\section{INTRODUCTION}

ASL is a completely noninvasive MRI method that measures brain perfusion by magnetically labeling blood in brain-feeding arteries by using a $180^{\circ} \mathrm{RF}$ pulse to invert the longitudinal magnetization of the spins in blood. The labeled blood traverses the vascular system and reaches the imaged slice where it exchanges with the tissue and thus contributes to the tissue magnetization. A standard EPI image (called a labeled image) is acquired at time TI milliseconds (inversion time) after the labeling. The EPI acquisition is repeated sufficiently long after the labeling to let the labeled blood fully relax. Therefore there are no effects of the previous labeling in this second EPI image (control image). The difference between the labeled and control images reflects the amount of the labeled blood that has perfused the tissue and can be used to image and quantify the tissue perfusion. ${ }^{1}$

The drawback of ASL is its sensitivity to noise. In general, the difference between the labeled and control images is around $1 \%-2 \%$ of the control image magnitude. A single control/label subtraction is therefore not sufficient for studying the perfusion and usually around 20-40 pairs of images need to be acquired to obtain reasonable signal-to-noise-ratio (SNR).

The ASL signal comes mainly from the gray matter tissue (GM). The perfusion of the white matter (WM) is on average approximately 3 times smaller than the gray matter perfusion in adults and almost 4 times smaller in children ${ }^{2}$. Also the $T_{1}$ relaxation of the GM and WM tissue is different ${ }^{3}$ and thus the perfusion signal in WM voxels decays more faster. The pixel size in ASL images is usually between $3 \times 3$ and $4 \times 4 \mathrm{~mm}$ and the slice thickness is between 5 and $8 \mathrm{~mm}$. The images are therefore severely affected by partial volume effects (PVE) as signal from gray and white matter is mixed in many voxels.

This makes quantification and denoising of ASL images complicated as apart from noise and local changes in perfusion there is also spatial signal variation due to tissue partial volume. Recently, Asllani, Borogovac and Brown ${ }^{4}$ have published an algorithm for correcting PVE in ASL images. The partial volume ratios are obtained from a high-resolution T1-weighted image by segmenting the WM, GM and cerebro-spinal fluid (CSF), see Figure 1. For every pixel, it is assumed that the tissue-specific perfusion is constant on a small square neighborhood. The different tissue contributions to the measured ASL signal are then estimated by linear 
a) upsampled ASL
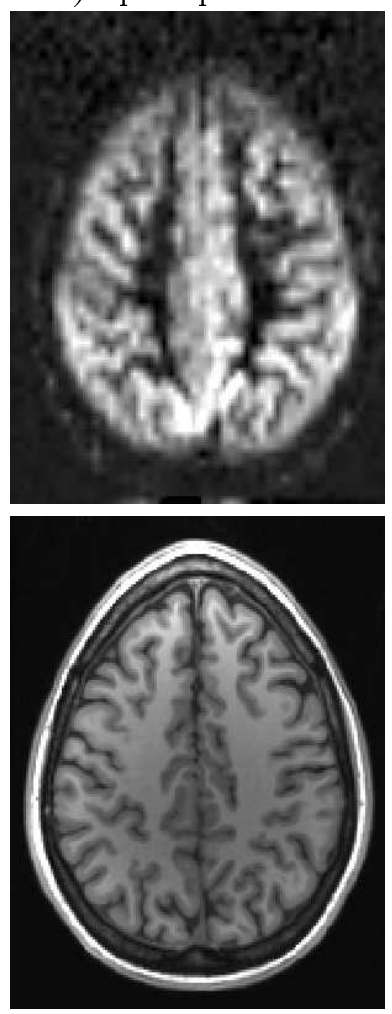

e) $T_{1}$ weighted image b) GM segmentation
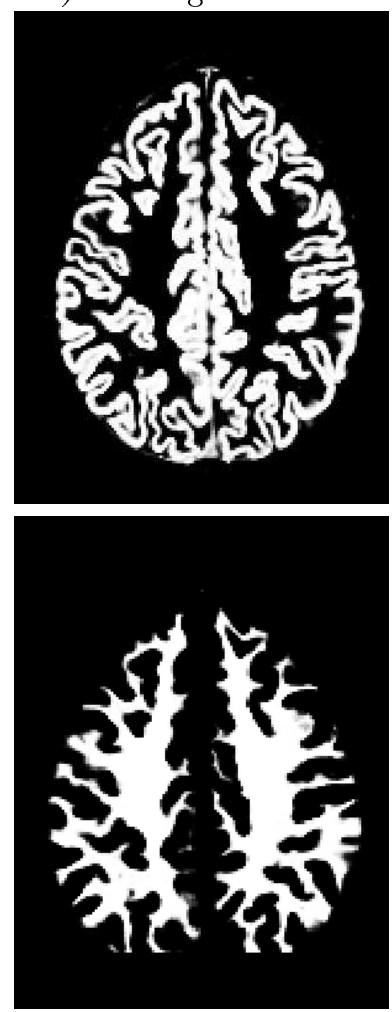

f) WM segmentation
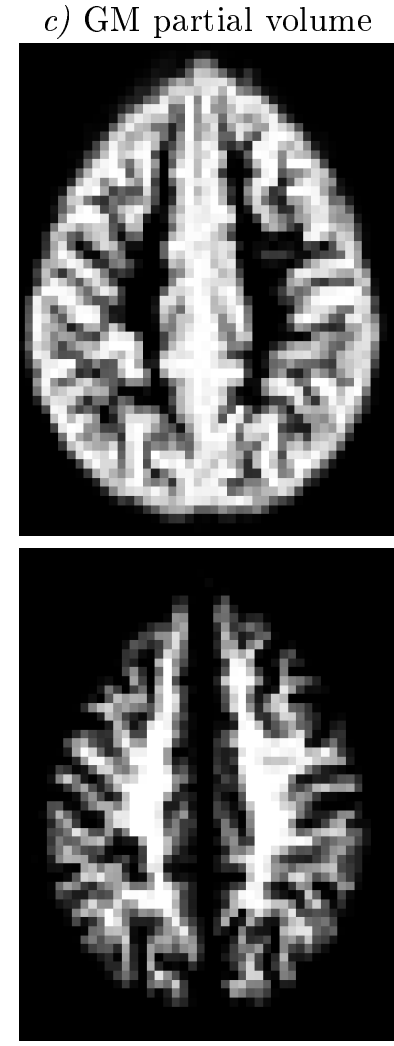

g) WM partial volume d) $\mathrm{ASL}$
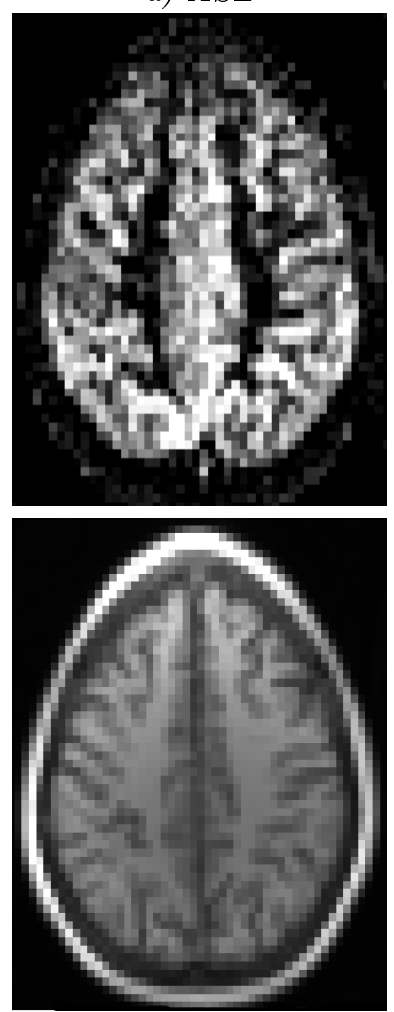

h) downsampled $T_{1}$ image

Figure 1. The $T_{1}$ weighted image $(f)$ is coregistered with the upsampled ASL perfusion-weighted image (a). The $T_{1}$ weighted image is segmented to obtain the GM $(b)$ and WM $(f)$ images. The segmented high resolution images are used to create the partial volume maps of WM $(g)$ and GM $(c)$ in the same resolution as the original ASL image (d). Note that the ASL signal is lower signal in the white matter than in the gray matter.

regression. This approach gives reasonable results and improves the cerebral blood flow (CBF) quantification which can be seriously underestimated or overestimated if the partial volume effects are disregarded. The sideeffect of this method is an inherent smoothing of the perfusion image with a square averaging filter.

The purpose of this article is to study and quantify this smoothing effect. An extension of the Asllani's algorithm is also proposed, where only the ratio between the GM and WM perfusion is estimated using the square averaging filter. The total variation of the overall perfusion is then minimized together while also minimizing the square difference between the measured values and values estimated using the GM/WM partial volumes and $\mathrm{WM} / \mathrm{GM}$ perfusion ratio. This way the perfusion maps are smoothed while the level of smoothing can be adapted to the noise level. It is shown that this approach produces better results than the original PVE correction method and it can be used to denoise the arterial spin labeling images. Validation is performed on a set of real 3T MRI images with 120 repetitions.

\section{METHOD(S)}

In the original Asllani's method the partial volume was corrected for both the difference and the control image in order to obtain the PVE corrected perfusion estimates of the continuous ASL. For the use with pulsed ASL, it is sufficient to use the part of the algorithm that corrects the labeled-control difference. ${ }^{5}$ The measured difference $\Delta M(r)$ in a pixel $r$ containing both GM, WM and CSF contributions is: ${ }^{4}$

$$
\Delta M(r)=P_{G M}(r) \Delta M_{G M}(r)+P_{W M}(r) \Delta M_{W M}(r)+P_{C S F}(r) \Delta M_{C S F}(r),
$$




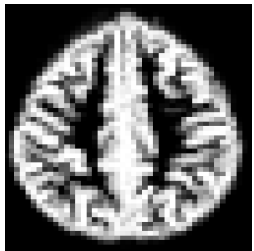

a) $P_{G M}$

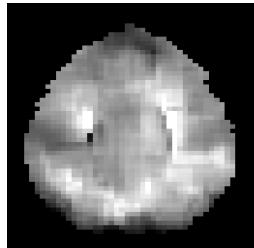

b) $\Delta M_{G M}$

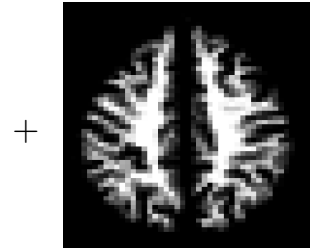

c) $P_{W M}$

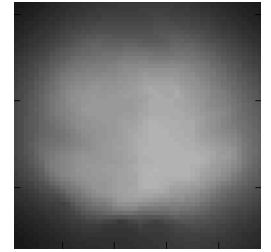

d) $\Delta M_{W M}$

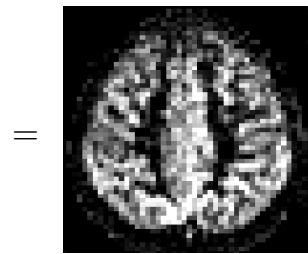

e) $\Delta_{M}$

Figure 2. The partial volume of $a$ ) gray and $c$ ) white matter is pixelwise multiplied with the $b, d$ ) tissue-specific difference magnetization and it should be equal with $e$ ) the measured control-label difference, see Eq. 1. The contribution of CSF is usually low or zero ${ }^{6}$ and thus it is not shown in this example.

where $P_{G M}, P_{W M}$ and $P_{C S F}$ are volume fractions of GM, WM and CSF; and $\Delta M_{G M}, \Delta M_{W M}, \Delta M_{C S F}$ are the values of tissue-specific magnetizations, see Figure 2.

Since there are three unknowns in Eq. 1 it cannot be solved in each pixel independently. It is therefore assumed that the difference magnetizations $\Delta M_{G M}, \Delta M_{W M}$ and $\Delta M_{C S F}$ are constant on a square $N \times N$ neighborhood centered around each pixel. From Equation 1, a system of $N^{2}$ linear equations with 3 variables is obtained for each pixel. Least-square solution of this system gives the tissue specific magnetizations for the pixel $r$.

The disadvantage of the Asllani's method is inherent averaging of the $\Delta M_{G M}$ values over the $N \times N$ neighborhood that can cause over-smoothing in the presence of significant spatial changes in the perfusion. To overcome this problem we propose a method that minimizes the total variation of the perfusion while also minimizing the squared difference of the measured and estimated values.

As a first step, the ratio of white matter/gray matter magnetization is estimated using the results of the Asllani's method

$$
R_{W M}(r)=\frac{\Delta M_{W M}}{\Delta M_{G M}} .
$$

The same ratio is computed also for CSF and then they are substituted into Eq. 1

$$
\begin{aligned}
& \Delta M(r)=P_{G M}(r) \Delta M_{G M}(r)+P_{W M}(r) \Delta M_{W M}(r) \frac{\Delta M_{G M}}{\Delta M_{G M}}+P_{C S F}(r) \Delta M_{C S F}(r) \frac{\Delta M_{G M}}{\Delta M_{G M}}, \\
& \Delta M(r)=P_{G M}(r) \Delta M_{G M}(r)+P_{W M}(r) R_{W M}(r) \Delta M_{G M}(r)+P_{C S F}(r) R_{C S F}(r) \Delta M_{G M}(r), \\
& \Delta M(r)=\Delta M_{G M}(r)\left[P_{G M}(r)+P_{W M}(r) R_{W M}(r)+P_{C S F}(r) R_{C S F}(r)\right], \\
& \Delta M(r)=\Delta M_{G M}(r) P_{t o t}(r),
\end{aligned}
$$

where $P_{t o t}$ is a sum of the tissue partial volumes which takes into account also the ratios of the tissue perfusions.

The perfusion map $\Delta M_{G M}$ is then smoothed by minimizing the total variation and data-fidelity term

$$
\min _{\Delta M_{G M}} \sum_{r}\left|\nabla\left(\Delta M_{G M}(r)\right)\right|+\lambda \sum_{r}\left|\Delta M(r)-\Delta M_{G M}(r) P_{t o t}(r)\right|^{2},
$$

where the parameter $\lambda$ controls the smoothing, see Figure 3. The error (Eq. 4) is then minimized using a primaldual gradient algorithm. ${ }^{7}$ With the knowledge of $\Delta M_{G M}$, the ratios of white matter/gray matter magnetization can be iteratively reestimated using Eq. 3 and Eq. 4 .

The optimal value of the parameter $\lambda$ that controls the smoothing was found experimentally and set to $\frac{1}{20 \sigma^{2}}$ where $\sigma$ is the mean standard deviation over all pixels computed using the sequence of all 120 repetitions (for the results for different values of $\lambda$ see Figure 4). 


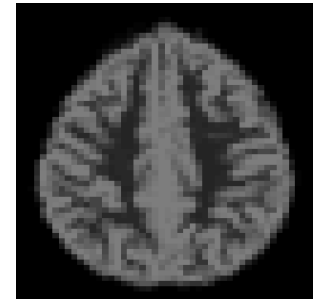

a) $P_{t o t}$

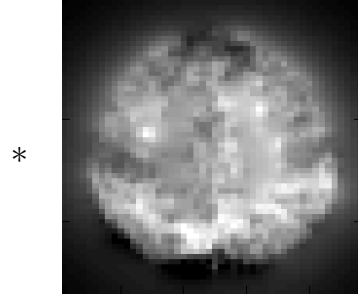

b) $\Delta M_{G M}$

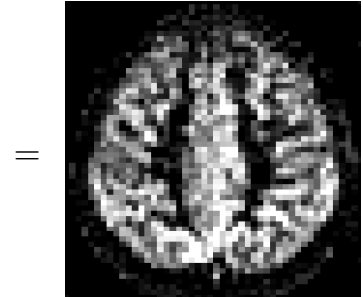

c) $\Delta_{M}$

Figure 3. Contrary to the PVE correction method by Asllani - only the $\Delta M_{G M}$ is estimated and smoothed in the proposed method using $P_{t o t}$ - a sum of the GM partial volume and the WM partial volume multiplied by the previously estimated gray/white matter perfusion ratio.
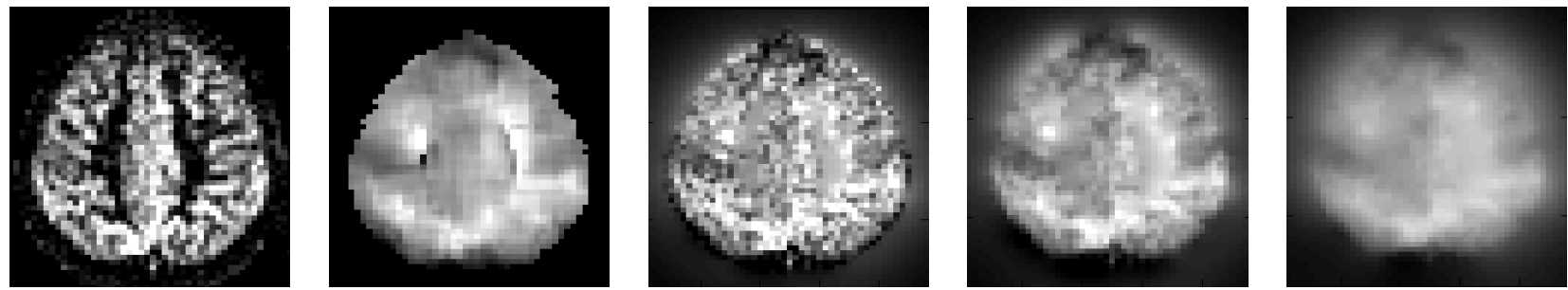

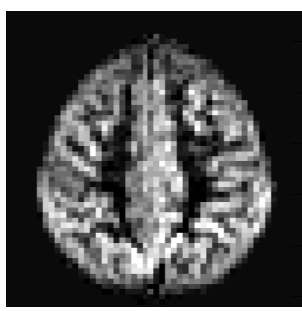

Original

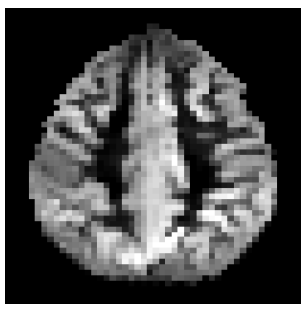

$3 \times 3$ average

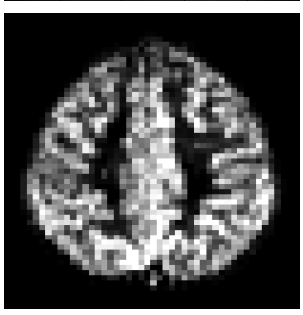

$\mathrm{TV}, \lambda=1 / \sigma^{2}$

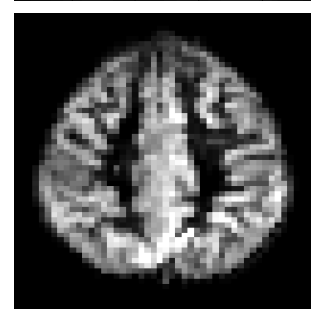

$\mathrm{TV}, \lambda=1 / 20 \sigma^{2}$

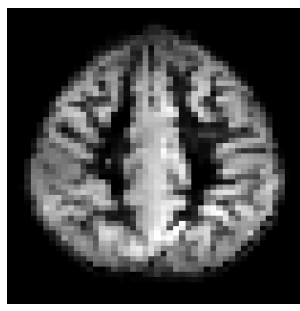

$\mathrm{TV}, \lambda=1 / 100 \sigma^{2}$

Figure 4. The ASL images with 20 and 120 repetitions are displayed in the leftmost column. In the top row of the four remaining columns is the estimated gray matter perfusion $\Delta M_{G M}$. The filtered perfusion image is in the bottom row. Results for the Asllani's method with $3 \times 3$ neighborhood and the results for the proposed method with varying parameter $\lambda$ are shown. 


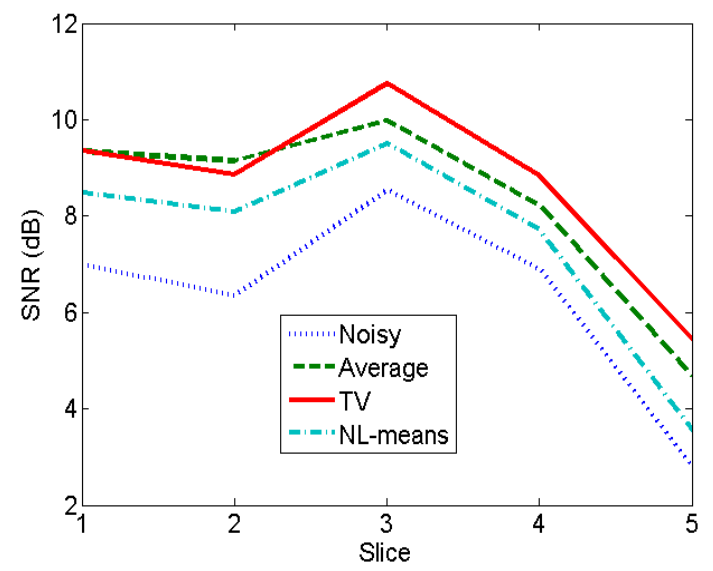

a) 15 repetitions

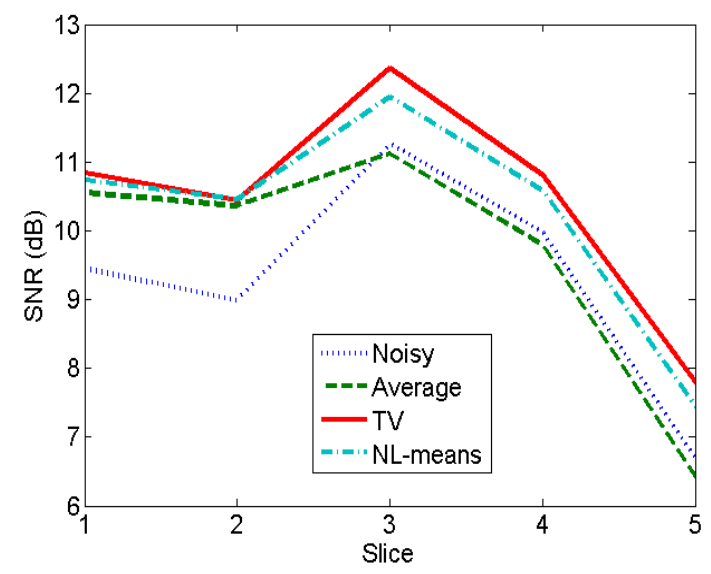

b) 30 repetitions

Figure 5. The results of denoising of the first dataset are displayed separately for all slices and for noisy input image consisting of a) 15 repetitions, b) 30 repetitions.

\subsection{Dataset and Experiments}

A healthy volunteer was scanned on Siemens Vario 3T MRI scanner (Siemens Medical Solutions, Erlangen, Germany) using the PICORE-QUIPSS II sequence with the following parameters: $T R=2500 \mathrm{~ms}, T E=17.7 \mathrm{~ms}$, flip angle $=90^{\circ}$, matrix size $=64 \times 64$, pixel size $=3 \times 3 \mathrm{~mm}$, SENSE factor $=2$, slice thickness $=8 \mathrm{~mm}$, inter-slice gap $=0.8 \mathrm{~mm}$, label width $=100 \mathrm{~mm}$, label $/$ slice gap $=30 \mathrm{~mm}$ and 120 repetitions (control-label pairs). Crusher gradients were used with the cutoff velocity set to $3 \mathrm{~cm} / \mathrm{s} .{ }^{8}$ Five axial slices were acquired in a sequential fashion from inferior to superior with inversion time $T I_{2}=1800 \mathrm{~ms}$ and QUIPSS saturation time $T I_{1}=700 \mathrm{~ms}$. A second dataset was acquired with the same parameters and $T R=3000 \mathrm{~ms}$. A high resolution T1-weighted scan of the whole head was also acquired using the gradient echo sequence: $T R=1900 \mathrm{~ms}, T E=2.98 \mathrm{~ms}$, flip angle $=9^{\circ}$, acquisition matrix $=256 \times 256 \times 182$ and voxel size $=1 \times 1 \times 1 \mathrm{~mm}$.

Each of the 120 repetitions was registered with the first using 3 D rigid transformation with mutual information criterion. ${ }^{9}$ The same criterion was employed for rigid-registration of the T1 image and the average control image upsampled to the resolution of the T1 image. The T1 image was segmented with the SPM8 toolbox and the results were downsampled to the ASL image resolution giving partial volume ratios of gray matter, white matter and cerebro-spinal fluid in each pixel.

The validation was done using leave-one-out cross validation. A set of the first $N$ volumes out of the 120 was used as a noisy input and an average of the remaining $120-N$ volumes as the ground truth. The same was repeated with the second set of $N$ images (from the $N+1 s$ t to the $2 N t$ h image) etc. The tissue perfusions were estimated using the Asllani's method with a $3 \times 3$ spatial neighborhood and also using the proposed method. The filtered image was created by multiplying the estimated tissue-specific perfusion with the corresponding tissue partial volumes. For comparison, the data were processed using the NL-means filter with a patch size of $3 \times 3$ pixels and the smoothing parameter set to $h=18 \sigma^{2}$ as proposed by Coupe, ${ }^{10}$ where 9 is the size of the patch and $\sigma$ is the noise standard deviation. The mean SNR of the $120 / N$ results was computed for each slice over pixels with the sum of GM, WM and CSF ratios exceeding 0.5 . The same process was repeated for $N=1,2,5$, $10,15,20,25$ and 30.

\section{RESULTS}

The results on the three most superior slices of the first dataset with 15 and 30 repetitions show the same relative performance of all tested methods. The PVE correction using total variation has $0.5 \mathrm{~dB}$ to $1.5 \mathrm{~dB}$ higher SNR than the other two methods. Whereas it has less than 0.3dB higher SNR than the Asllani's method on the first two slices with 30 repetitions and even up to 0.3dB lower SNR for the second slice with 15 repetitions. 
a) Noisy
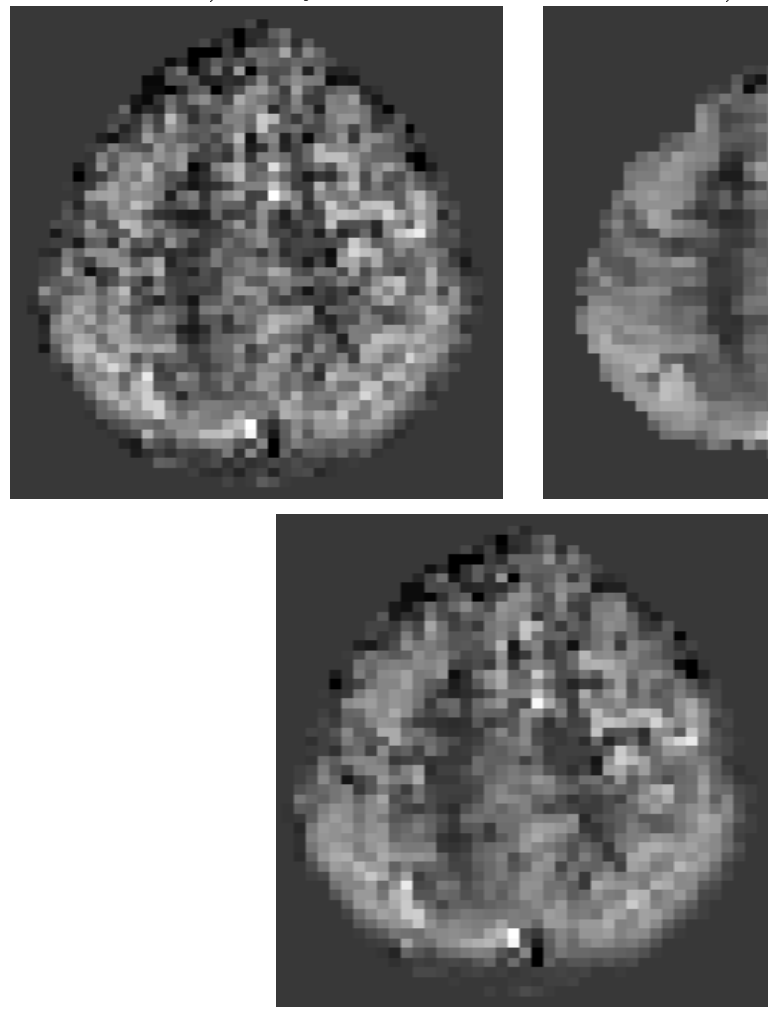

d) NL-means b) Average
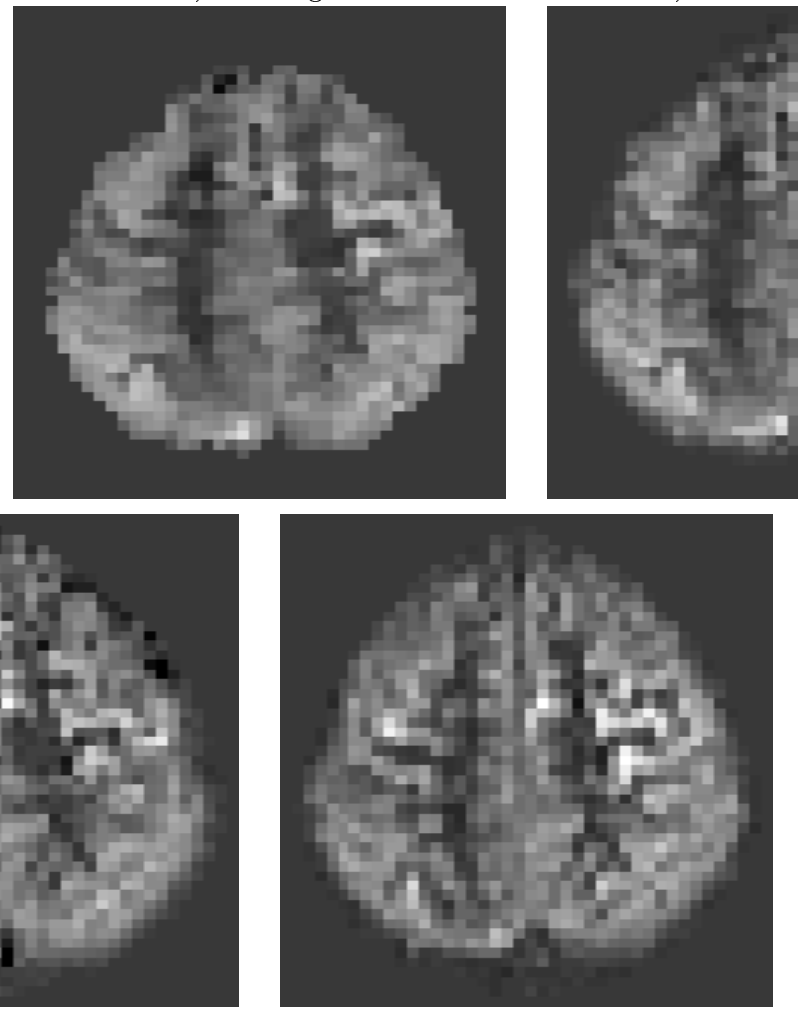

e) Ground truth c) Total variation

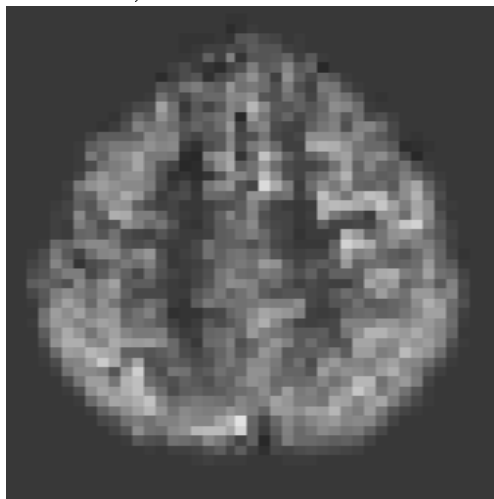

Figure 6. The average of 10 repetitions of the fourth slice (from inferior to superior) is displayed (a). The filtering using Asllani's method (b), its extension using total variation (c) and NL-means (d). The average over all 120 repetitions is taken as the ground truth image (e).

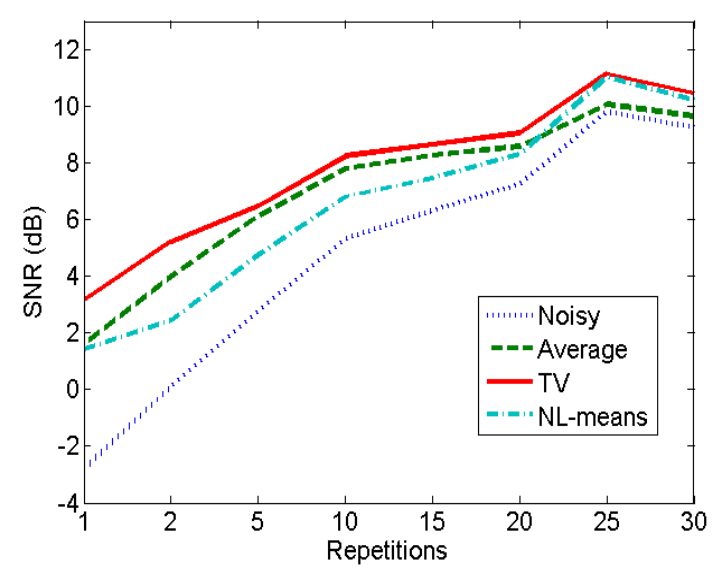

a) Dataset 1

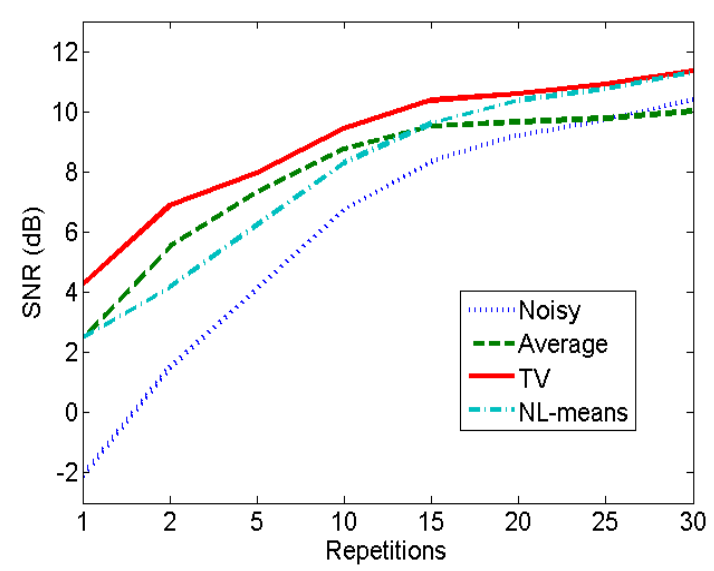

b) Dataset 2

Figure 7. The mean SNR over all slices is displayed for $a$ ) the first and $b$ ) the second dataset. 
a) Noisy

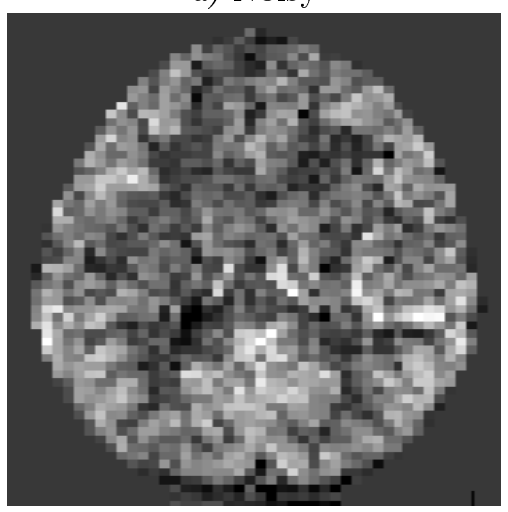

b) Average

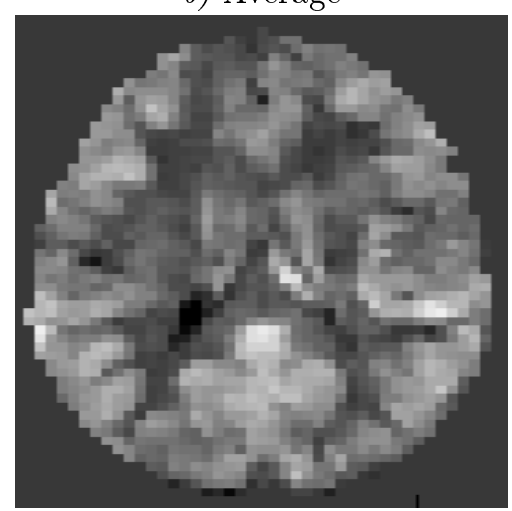

c) Total variation

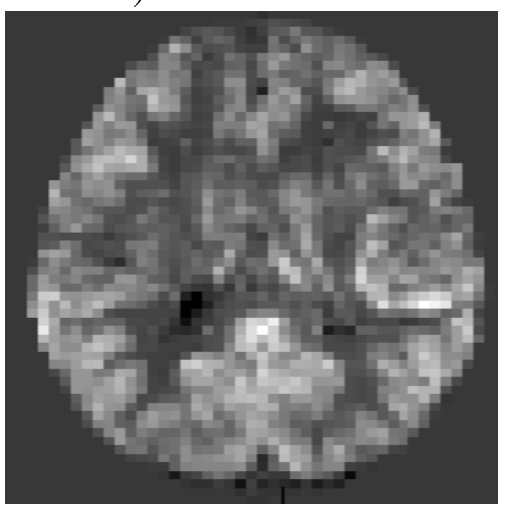

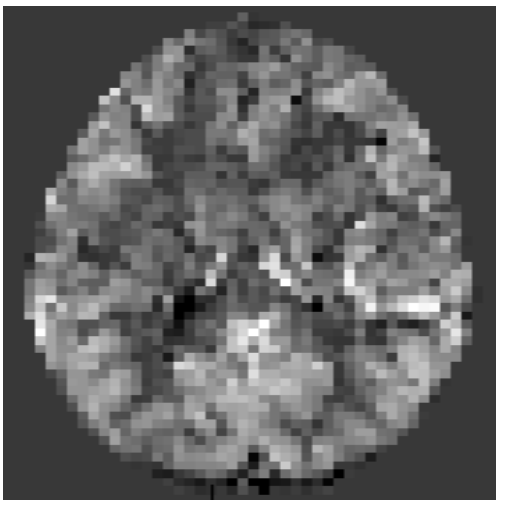

d) NL-means

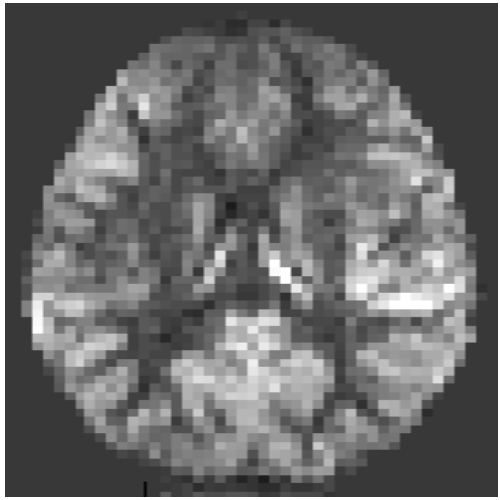

e) Ground truth

Figure 8. The filtering results for the first slice of the first dataset and an input image with 15 repetitions.

The overall results for all slices for increasing number of repetitions show that the proposed method has 0.5 to $2 \mathrm{~dB}$ higher SNR than both the other methods for images with less than 20 repetitions. For more repetitions, the performance of the method using $3 \times 3$ neighborhood is decreasing. However, the NL-means filtering is within $0.3 \mathrm{~dB}$ from the total variation approach.

The results of filtering on images with 10,15 and 20 repetitions are displayed for qualitative comparison, see Figures 6,8 and 9 . The results from $3 \times 3$ neighborhood averaging method are clearly oversmoothed in all cases. Whereas the total variation method presents clear decrease of noise without oversmoothing the structures. There is considerably more noise in the NL-means filtered images than in the images filtered by the proposed method for images with 10 and 15 repetitions. However, the noise level is similar for the image with 20 repetitions.

\section{DISCUSSION AND CONCLUSIONS}

We have proposed an extension of the PVE correction algorithm ${ }^{4}$ by minimizing the total variation of tissuespecific difference magnetization and we have used this method to denoise ASL images. The results are compared with those of the original method with $3 \times 3$ neighborhood averaging and with NL-means filtering.

The PVE correction method using the $3 \times 3$ neighborhood clearly oversmooths the image as can be seen for all images with 10, 15 and 20 repetitions. Nevertheless the SNR is increased in all cases for input images with up to 20 repetitions. However, for images with more than 20 repetitions a clear decrease of SNR can be noticed, see Figure 5b. The oversmoothing is less significant on the two most inferior slices. This is because there are large homogeneous regions of gray and white matter where the oversmoothing is not so evident. As a consequence of this, there is higher SNR than that of the total variation method on the two inferior slices with 15 repetitions, see Figure 5a. On the contrary for images with more than 20 repetitions the performance of the Asllani's method is decreasing and the filtered result has even lower SNR than the input images. 
a) Noisy

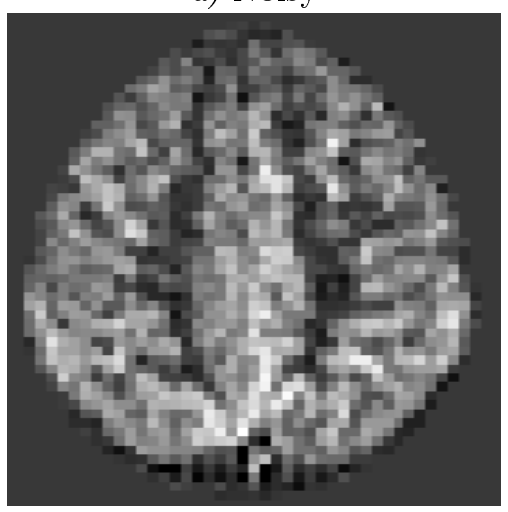

b) Average

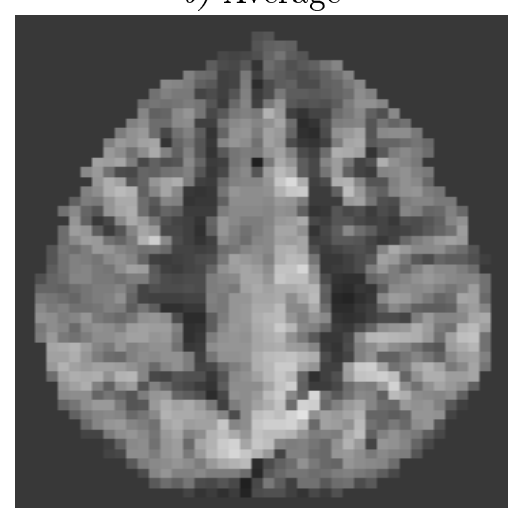

c) Total variation

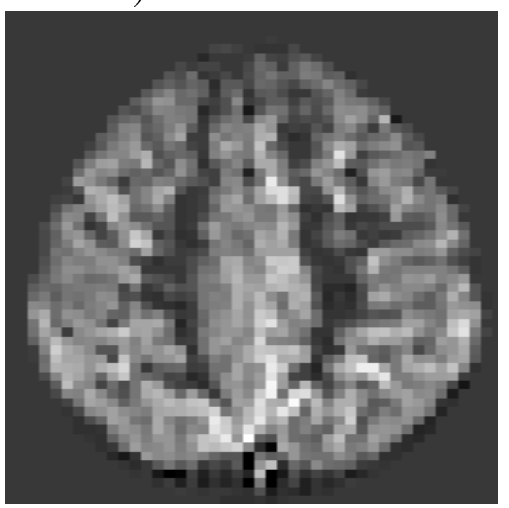

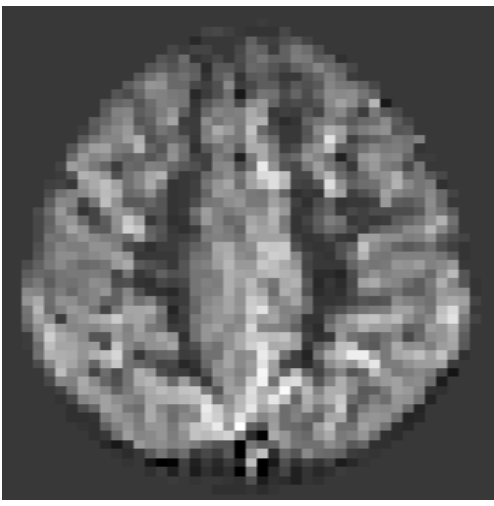

d) NL-means

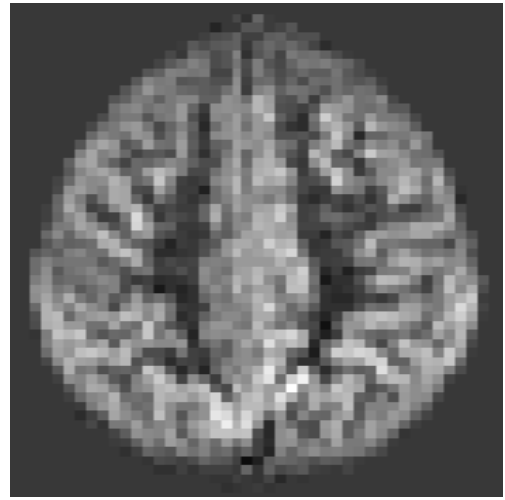

e) Ground truth

Figure 9. The results for the third slice of the first dataset and an input image with 20 repetitions.

The NL-means filtering without any prior knowledge of the perfusion gives the worst results for noisy images with less than 15 repetitions. However, as the quality of the images rises the knowledge of partial volumes becomes less advantageous and the NL-means SNR is getting close to the results of the proposed method.

Nevertheless, the proposed TV-filtration improves the SNR in almost all tested slices and noise levels showing the ability of the method to filter the noise in ASL images without mixing the GM and WM regions. The advantage of the TV filtration over the simple average filtration is that it is more flexible to changes in perfusion and does not therefore smooth out regional variations of perfusion. This makes it more suitable for diseased patients, where anomalies in perfusion need to be detected.

\section{REFERENCES}

[1] Parkes, L. M. and Detre, J. A., [Quantitative MRI of the brain: Measuring changes caused by disease], ch. ASL: Blood perfusion measurements using arterial spin labelling, 455-473, John Wiley \& Sons (2003).

[2] Biagi, L., Abbruzzese, A., Bianchi, M. C., Alsop, D. C., Guerra, A. D., and Tosetti, M., "Age dependence of cerebral perfusion assessed by magnetic resonance continuous arterial spin labeling," Journal of Magnetic Resonance Imaging 25, 696-702 (2007).

[3] Alsop, D. C. and Detre, J. A., "Reduced transit-time sensitivity in noninvasive magnetic resonance imaging of human cerebral blood flow," Journal of Cerebral Blood Flow Metabolism 16, 1236-49 (November 1996).

[4] Asllani, I., Borogovac, A., and Brown, T. R., "Regression algorithm correcting for partial volume effects in arterial spin labeling mri," Magnetic Resonance in Medicine 60, 1362-1371 (2008).

[5] Barbier, E. L., Lamalle, L., and Décorps, M., "Methodology of brain perfusion imaging," Journal of Magnetic Resonance Imaging 13, 496-520 (2001). 
[6] X. Golay, J. Hendrikse, T. C. L., "Perfusion imaging using arterial spin labeling," Topics in Magnetic Resonance Imaging 15, 10-27 (February 2004).

[7] Zhu, M. and Chan, T., "An efficient primal-dual hybrid gradient algorithm for total variation image restoration," tech. rep., UCLA CAM Report 08-34 (2008).

[8] Ye, F. Q., Mattay, V. S., Jezzard, P., Frank, J. A., Weinberger, D. R., and McLaughlin, A. C., "Correction for vascular artifacts in cerebral blood flow values measured by using arterial spin tagging techniques," Magnetic Resonance in Medicine 37(2), 226-235 (1997).

[9] Maes, F., Collignon, A., Vandermuelen, D., Marchal, G., and Suetens, P., "Multimodality image registration by maximization of mutual information," IEEE Transactions on Medical Imaging 16, 187-198 (April 1997).

[10] Coupé, P., Yger, P., Prima, S., Hellier, P., Kervrann, C., and Barillot, C., "An optimized blockwise non local means denoising filter for 3D magnetic resonance images," IEEE Transactions on Medical Imaging 27, 425-441 (April 2008).

[11] Gallichan, D. and Jezzard, P., "Variation in the shape of pulsed arterial spin labeling kinetic curves across the healthy human brain and its implications for CBF quantification," Magnetic Resonance in Medicine 61(3), 686-695 (2009). 